\title{
Hyperthermia and postmortem biochemical investigations
}

\author{
Cristian Palmiere $\cdot$ Patrice Mangin
}

Received: 2 March 2012 / Accepted: 24 May 2012 / Published online: 5 June 2012

(C) Springer-Verlag 2012

\begin{abstract}
The postmortem diagnosis of heat-related deaths presents certain difficulties. Firstly, preterminal or terminal body temperatures are often not available. Additionally, macroscopic and microscopic findings are nonspecific or inconclusive and depend on survival duration after exposure. The diagnosis of hyperthermia is therefore essentially based on scene investigation, the circumstances of death, and the reasonable exclusion of other causes of death. Immunohistochemistry and postmortem biochemical investigations have been performed by several authors in order to better circumstantiate the physiopathology of hyperthermia and provide further information to confirm or exclude a heat-related cause of death. Biochemical markers, such as electrolytes, hormones, blood proteins, enzymes, and neurotransmitters, have been analyzed in blood and other biological fluids to improve the diagnostic potential of autopsy, histology, and immunohistochemistry. The aim of this article is to present a review of the medicolegal literature pertaining to the postmortem biochemical investigations that are associated with heat-related deaths.
\end{abstract}

Keywords Hyperthermia $\cdot$ Forensic pathology $\cdot$ Postmortem diagnosis $\cdot$ Postmortem biochemistry

C. Palmiere $(\triangle)$

University Centre of Legal Medicine,

Rue du Bugnon 21,

1011 Lausanne, Switzerland

e-mail: cristian.palmiere@chuv.ch

P. Mangin

University Centre of Legal Medicine,

Rue Michel-Servet 1,

1211 Genève, Switzerland

\section{Introduction}

Hyperthermia is clinically diagnosed by a core temperature $>40{ }^{\circ} \mathrm{C}\left(104{ }^{\circ} \mathrm{F}\right)$, occurring when the body's thermoregulatory mechanisms are no longer capable of effectively dissipating heat. Hyperthermia is distinguished from fever and hyperpyrexia which characteristically occur with an increased hypothalamic set point due to severe infection or central nervous system hemorrhages.

Hyperthermia is more often observed in the summer months in particularly hot environments and is more common in the very young and the elderly as well as those presenting severe preexisting medical conditions [1,2]. Indeed, a significant number of heat-related deaths occurring during a heat wave do not result solely from hyperthermia but from heat stress, which fatally exacerbates any important, underlying medical disease [3-8].

The elderly, children, and those suffering from cardiovascular disease are at greater risk of heat-related death. Predisposing factors that may limit the body's ability to emplace an effective thermoregulatory response to excessive heat include dementia, psychiatric illnesses, diabetes, hyperthyroidism, obesity, burns, alcohol consumption, and chronic alcohol misuse. These conditions complicate the usual dissipation of heat due to increased metabolism, decreased activity, increased subcutaneous fat, or the inability to peripherally vasodilate, sweat properly, and release excessive heat $[1,2,9,10]$.

Bunai et al. [11] described a case of fatal hyperthermia that occurred in a 39-year-old man with a body mass index of 31.4 and a state of excited delirium during a police arrest. Siegler [12] reported a case of fatal heat stroke in a young woman with previously undiagnosed Hashimoto's thyroiditis. According to the author, in conditions of high temperature, hypothyroid patients continue to conserve heat or 
have attenuated heat loss rather than heat dissipation through sweating. Moreover, the decreased adrenergic activity in hypothyroidism would lead to decreased cardiac output, again interfering with effective heat loss.

Dementia and psychiatric illnesses can also compromise the body's ability to adequately react to a hot environment. Hyperthermia (exertional heat stroke) can also be fatal in people participating in strenuous outdoor physical activities in hot weather (e.g., athletes, military personnel, and workers) and especially in people with severe, preexisting medical conditions [13-26]. Voluntary exposure to extreme dry or humid heat may result in illness or even death by heat stroke and/or dehydration [27].

Social and environmental factors, such as self-care incompetence and social isolation, may predispose some people to fatal hyperthermia. Infants and young children are more susceptible to developing hyperthermia from a hot environment given their reduced sweating capacity, higher metabolic rate, greater heat vulnerability, and larger body surface-to-volume ratio than adults $[1,2]$.

Most heat-related deaths are due to weather conditions. Hyperthermia deaths in children younger than 4 years of age often coincide with vehicular entrapment on warm days. Death can occur in a relatively short amount of time in these cases [28-40]. Only a small percentage of heat-related deaths are of man-made origin (e.g., in boiler rooms, furnaces, vehicles, or factory-generated extreme heat conditions or electrical appliance misuse) [41-43].

Various classes of licit and illicit drugs can increase the risk of developing fatal hyperthermia. Drugs with anticholinergic side effects (e.g., tricyclic antidepressants, antihistamines, antiparkinsonians, and antipsychotics) impair the sweating mechanism and hinder the body's ability to eliminate heat properly. The diuretics often used by the elderly cause volume depletion, thereby limiting the body's ability to increase cardiac output and perspire $[1,2]$.

Mild to severe hyperthermia may be associated with recreational or pharmaceutical sympathomimetic drug use. Amphetamine and methamphetamine augment the release of norepinephrine, dopamine, and serotonin from presynaptic nerve terminals and inhibit the synaptic uptake. Some amphetamine metabolites inhibit monoamine oxidase. Although structurally dissimilar, the clinical effects of amphetamine and methamphetamine are nearly identical to cocaine. The central thermoregulatory disturbances from sympathomimetic drugs arise from complex interactions between dopamine and serotonin in the brain stem and hypothalamus and can result in an increased rate of metabolism and core temperature. Peripherally, sympathomimetics disrupt temperature homeostasis by several pathways and can result in the vasoconstriction of peripheral vessels and impaired heat dissipation [44].

Neuroleptic malignant syndrome (NMS) is a rare but potentially life-threatening side effect of psychotropic medications that have antidopaminergic action. NMS may be generated by either an idiosyncratic response to dopamine antagonists or by dopamine agonist withdrawal. The predictive signs of NMS have historically included muscular rigidity and changes in mental status, followed by hyperthermia (temperature $>105^{\circ} \mathrm{F}$ ) and autonomic instability. The pathophysiological mechanism of NMS is thought to involve the blockage of dopaminergic transmission in the nigrostriatal pathway [45-51].

Malignant hyperthermia (MH) is a skeletal muscle pharmacogenetic disorder characterized by a hypermetabolic response to potent, volatile anesthetic gases, such as halothane, sevoflurane, and desflurane, as well as to the depolarizing muscle relaxant succinylcholine. The body's reactions to these drugs are dominated by progressive hyperthermia, hence the name. The triggering agents cause an increase in the concentration of free cytoplasmic calcium, which is released from the sarcoplasmic reticulum stores via the muscle ryanodine receptor. The ryanodine receptor $(R Y R I)$ gene encodes the key channel that mediates the calcium release in skeletal muscle during excitation-contraction coupling. Mutations in the ryanodine receptor gene are attributed to approximately $50 \%$ of patients susceptible to MH [52-55].

Serotonin syndrome is a condition characterized by central serotonin hyperstimulation. Classic findings of severe serotonin syndrome include hyperthermia, mental status changes, autonomic instability, and altered muscle tone or rigidity. A number of medications have been implicated in the induction of serotonin syndrome, including those that reduce metabolism (e.g., monoamine oxidase inhibitors), increase production (e.g., L-tryptophan), or inhibit serotonin uptake (e.g., fluoxetine, clomipramine, meperidine, dextromethorphan, pentazocine, and fenfluramine). The complications resulting from hyperthermia associated with severe serotonin syndrome may include rhabdomyolysis, cardiac dysrhythmias, renal failure, disseminated intravascular coagulation, seizures, coma, and death [56-60].

The postmortem diagnosis of heat-related death presents certain difficulties. Although people dying of hyperthermia can have body temperatures $>40.6^{\circ} \mathrm{C}\left(105^{\circ} \mathrm{F}\right)$, in routine forensic pathology, preterminal or terminal body temperatures are often not available. Autopsy findings may be minimal and difficult to interpret, especially if bodies have reached advanced stages of decomposition in a hot environment. Thus, the diagnosis of hyperthermia is mainly based on scene investigation and the circumstances of death [1, 2, 61].

One significant difficulty faced by forensic pathologists in the past was the lack of uniform diagnostic criteria for heat-related deaths. In response to this problem, the National Association of Medical Examiners' Ad Hoc Committee on the Definition of Heat-Related Fatalities recommended that a death be designated as heat related if exposure to high ambient temperature either caused or significantly contributed to death. The committee also recommended that the 
diagnosis of heat-related death be based on a history of exposure to high environmental temperature as determined by investigation and the reasonable exclusion of other causes of death.

Heat-related death should be listed as a possible cause of death or at least a significant contributing condition when the antemortem body temperature at the time of death cannot be established and the circumstances of the death suggest hyperthermia. A heat-related cause of death may furthermore be assumed if the investigation provides compelling evidence of continuous exposure to a hot environment and fails to identify an independent cause of death. The collection of blood, urine, vitreous humor, and other biological fluids for toxicological and biochemical investigations is strongly suggested since autopsy findings in heatrelated deaths are nonspecific. Nonetheless, a diagnosis of heat-related death is still principally based on investigative information [61, 62].

\section{Autopsy, histology, and immunohistochemistry}

Necropsy findings in cases of fatal heat stroke are nonspecific and depend on the survival duration after exposure. Tissue damage is caused by several mechanisms including ischemia as a result of massive blood shunting away from the internal organs to the skin. When victims of heat stroke survive $<12 \mathrm{~h}$, the only abnormalities noted may be petechial or larger hemorrhages in the skin with slippage or petechial hemorrhages over the surface of the lungs, heart, and serosal surfaces. Pulmonary and cerebral edema with diffuse neuronal injury may be observed following longer periods of survival $[1,10,42,61,63]$.

Right-sided cardiac dilatation, subendocardial hemorrhage of the left ventricle, visceral congestion (particularly in the lungs and the brain), and petechial hemorrhages in the thymus, the perifollicular area of the spleen, and lymph nodes have also been described in people who died shortly after the onset of heat stress [11, 43]. Tissue damage may be revealed by centrilobular necrosis and midzonal vacuolar changes in the liver, neuronal degeneration of the brain, and acute pancreatitis. Rhabdomyolysis may result in acute tubular necrosis of the kidneys. Should disseminated intravascular coagulation occur, fibrin thrombi may appear in small blood vessels [61]. Other microscopic findings, such as pulmonary fat embolization, have also been described [64].

Fineschi et al. [42] studied the tissue expression of the heat shock proteins HSP 70, 27, and 90 in a case of heat stroke involving an infant who died in an incubator. They found that the expression of these proteins was increased in the epithelium of the trachea and in the skin.

Quan et al. [65] observed high ubiquitin immunoreactivity in the midbrain periaqueductal gray matter in cases of hyperthermia and hypothesized that the neuronal ubiquitination may be a finding of nonspecific neurodegeneration as a consequence of metabolic disorders or central nervous system dysfunction, depending on the survival time. Ishikawa et al. [66] found low myoglobin and ubiquitin immunopositivity in the renal tubular epithelium cells of hyperthermia fatalities and postulated that this result may be attributed to advanced renal tubular damage (despite advanced skeletal muscle damage) and the downregulation of ubiquitin-dependent protein degradation, as previously suggested by Smith et al. [67]. A markedly low pituitary adrenocorticotropic hormone (ACTH) immunopositivity in hyperthermia cases has been described by Ishikawa et al. [68], which was likely related to multiple organ dysfunction. The same authors also observed [69] a significantly decreased rate of neuronal dopamine immunopositivity in the hypothalamus and increased noradrenaline and dopamine immunopositivity rates in the adrenal medulla. According to the authors, these findings suggested that there was a terminal hyperfunction of the sympathetic/adrenomedullary system involving the adrenal medulla in fatal hyperthermia. They concluded that noradrenaline and dopamine immunopositivity in the adrenal medulla may be used as markers to investigate the magnitude of the physical stress responses involving the sympathetic/adrenomedullary system in the hyperthermia death process. Conversely, the lower rate of neuronal dopamine immunopositivity in the hypothalamus in hyperthermia could indicate a dysfunction of the central nervous system, which appeared to be independent of the activation of the sympathetic/adrenomedullary system, as demonstrated by increased serum and cerebrospinal fluid (CSF) catecholamine levels. According to the authors, such hypothalamic dysfunction could lead to deterioration of the hypothalamic-pituitary-adrenal stress response system, hence concluding that the hypothalamic neuronal dopamine immunopositivity might be used as a marker to investigate the function of the hypothalamic-pituitary-adrenal axis. Finally, Yoshida et al. [70] described a high hypothalamic neuronal chromogranin A ( $\mathrm{CgA})$ immunopositivity in hyperthermia cases, together with no increase in $\mathrm{CgA}$ immunopositivity in the adrenal medulla.

\section{Postmortem biochemistry}

\section{Electrolytes}

The physiopathology of hyperthermia as a consequence of heat stroke typically involves dehydration and electrolyte disturbance $[1,71]$. Abnormalities in antemortem serum sodium concentrations are traditionally considered as reflected in postmortem vitreous humor values, making it possible to diagnose hypo- or hypernatremia at the time of death. Similar to sodium, vitreous chloride concentrations have been determined to be 
relatively stable during the early postmortem period, and abnormalities in antemortem serum chloride are thought to be reflected in postmortem vitreous values [72-80]. In a study performed on 385 medicolegal autopsy cases, Li et al. [81] analyzed postmortem calcium and magnesium levels in pericardial fluid. When compared with other studied groups, hyperthermia cases had lower calcium concentrations, likely related to skeletal muscle damage, higher magnesium levels, and higher $\mathrm{Mg} / \mathrm{Ca}$ ratios.

\section{Catecholamines}

Kortelainen et al. [82] investigated urinary catecholamine levels in five hyperthermia cases (four of them under the influence of ethanol) and compared these values with those from a control group of five sudden violent deaths. The authors observed increased urinary noradrenaline levels in hyperthermia victims, whereas adrenaline and dopamine concentrations were not significantly different between the two groups. The authors assumed that high postmortem urinary noradrenaline levels in hyperthermia victims could represent a sign of sympathetic hyperactivity following heat exposure. Thus, they concluded that urinary noradrenaline levels could be used as a biochemical marker in investigations of hyperthermia fatalities. Zhu et al. [83] analyzed catecholamine levels (adrenaline, noradrenaline, and dopamine) in 542 forensic autopsy cases in postmortem serum from different sampling sites (left and right heart chambers, and subclavian and external iliac vein). The characteristic findings of hyperthermia fatalities were higher dopamine levels in both cardiac and peripheral blood, accompanied by significantly higher adrenaline and noradrenaline levels in external iliac venous blood. According to the authors, these findings suggested that postmortem serum catecholamine levels reflected the magnitude of physical stress responses during the death process in individual cases.

In a successive study, Ishikawa et al. [69] observed elevated serum and CSF catecholamine levels in hyperthermia cases, together with a lower neuronal dopamine immunopositivity in the hypothalamus. According to the authors, these findings suggested a dysfunction of the hypothalamus in hyperthermia fatalities, which appeared to be independent of the activation of the sympathetic/adrenomedullary system, as shown by the increased serum and CSF catecholamine levels.

\section{Pituitary hormones}

Ishikawa et al. [68, 84] investigated ACTH and growth hormone $(\mathrm{GH})$ levels in postmortem serum from right heart blood and CSF in two series of forensic autopsies including hyperthermia fatalities. In an initial study performed on 162 forensic autopsies [68], the authors observed that hyperthermia cases presented postmortem serum and CSF ACTH levels that were significantly lower than reference values and concentrations found in other studied groups, in which CSF levels were usually much higher than the postmortem serum levels. Additionally, serum and CSF ACTH levels were significantly correlated. Hyperthermia fatalities also displayed a lower CSF/serum ratio and a markedly low pituitary ACTH immunopositivity, which was likely related to multiple organ dysfunction due to hyperthermia. In a successive study performed on 116 forensic autopsies that focused on postmortem serum and CSF GH levels and pituitary GH immunopositivity [84], hyperthermia fatalities did not display peculiar changes, also suggesting that the postmortem serum and CSF GH and ACTH levels behave differently based on the varied stress reactions of the hypothalamic-pituitary-adrenal axis.

\section{Markers of renal function}

Zhu et al. [85-87] analyzed urea nitrogen, creatinine, and uric acid levels in pericardial fluid and postmortem serum from different sampling sites (right heart, left heart, subclavian vein, and external iliac vein). In an initial study [85] performed on 395 medicolegal autopsy cases, heat stroke fatalities displayed increased postmortem serum creatinine levels along with normal urea nitrogen concentrations, which was likely related to skeletal muscle damage due to hyperthermia. These findings, together with the fact that myoglobinuria was often observed in cases of death by heat stroke, suggested that the elevation of postmortem serum creatinine levels was caused by mild muscle damage that was antecedent to myoglobinemia and myoglobinuria. In another study [86] performed on 409 medicolegal autopsy cases, the authors examined urea nitrogen, creatinine, and uric acid pericardial fluid levels. Hyperthermia cases presented mild increases in urea nitrogen concentrations, mild to moderate elevations in creatinine values, a low urea nitrogen/creatinine ratio $(<10)$, and moderate elevations in uric acid levels. Lastly, in a third study [87] performed on 556 medicolegal autopsy cases, the authors compared the differences between pericardial fluid and postmortem serum levels of urea nitrogen, creatinine, and uric acid. Postmortem serum was obtained from different sampling sites (the right heart, left heart, and external iliac vein). The authors observed similar distributions in postmortem serum and pericardial fluid for all markers in hyperthermia fatalities. However, some of these cases presented postmortem serum creatinine levels that were markedly higher than pericardial fluid levels. Significant changes in pericardial fluid levels became apparent several hours later than in serum, leading the authors to conclude that elevations in pericardial fluid urea nitrogen, creatinine, and/or uric acid levels could suggest prolonged survival over hours. 


\section{Markers of cardiac function}

Zhu et al. [88-91] and Wang et al. [92] investigated a series of cardiac markers, including atrial natriuretic peptide (ANP), brain natriuretic peptide (BNP), cardiac troponin $\mathrm{T}$ (cTnT), cardiac troponin I (cTnI), creatine kinase MB (CK-MB), and myoglobin $(\mathrm{Mb})$ in blood and pericardial and cerebrospinal fluids. In a study [88] performed on 405 medicolegal autopsy cases, the authors analyzed cTnT levels in pericardial fluid and postmortem serum from different sampling sites (right heart, left heart, and external iliac vein). cTnT levels in postmortem serum from heart blood and pericardial fluid were higher than those in peripheral blood. Furthermore, hyperthermia cases had significantly higher postmortem serum cTnT levels at each site and especially high pericardial fluid cTnT levels. The morphological severity of myocardial damage was well correlated with cTnT levels in postmortem serum from heart blood and pericardial fluid. Thus, cases that showed diffuse myocardial damage were also characterized by the highest cTnT levels. Analyses with regard to the time since death revealed that significantly high cTnT levels were observed in both early $(<12 \mathrm{~h})$ and late $(12-48 \mathrm{~h})$ postmortem periods. Finally, comparisons with histopathological findings suggested that advanced myocardial damage involving swelling and liquefactive necrosis were closely related to increased cTnT levels. In a study performed on 263 forensic autopsy cases, Zhu et al. [90] analyzed ANP, BNP, and cTnT pericardial fluid levels. The results of this study revealed that there was no significant postmortem influence on pericardial ANP and BNP levels within $72 \mathrm{~h}$ after death. Additionally, ANP and BNP were negatively correlated with cTnT levels in pericardial fluid, which suggested that their production by cardiac tissue was reduced depending on the severity of myocardial damage. Hyperthermia fatalities displayed decreased pericardial ANP and BNP levels. Since previous findings by the same authors had shown increased postmortem serum and pericardial cTnT levels in hyperthermia fatalities, the authors concluded that massive myocardial damage due to advanced hypoxia was responsible for high cTnT and low ANP and BNP levels in cases of heat stroke. In a study performed on 234 forensic autopsy cases, the authors [91] investigated cTnT, cTnI, and CK-MB levels in pericardial fluid and postmortem serum from different sampling sites (right heart, left heart, and external iliac vein). Findings for $\mathrm{cTnI}$ were similar to those for $\mathrm{cTnT}$ previously described by the same authors. cTnI levels in postmortem serum from cardiac blood and pericardial fluid in hyperthermia cases were relatively high, whereas cTnI levels were not significant high for postmortem serum from peripheral blood. Increased cTnI levels in postmortem serum from cardiac blood and pericardial fluid were related to the morphological severity of myocardial damage, which was due to diffuse liquefactive necrosis, whereas CKMB levels were not dependent on the morphological severity of myocardial damage. Wang et al. [92] investigated CK-MB, $\mathrm{cTnI}$, and $\mathrm{Mb}$ levels in pericardial and cerebrospinal fluids and compared them with those obtained from right heart blood postmortem serum. The study was performed on 295 forensic autopsy cases. The authors found that hyperthermia fatalities were characterized by significantly higher $\mathrm{Mb}$ levels in pericardial and cerebrospinal fluids. According to the authors, these findings indicated that advanced skeletal muscle damage in fatal hyperthermia affected CSF. Additionally, the results confirmed the usefulness of combined determinations of biochemical markers in several biological fluids for evaluating the severity of myocardial and skeletal muscle damage in the death process.

\section{$\mathrm{CgA}$, serotonin, and $\mathrm{S} 100 \mathrm{~B}$}

Yoshida et al. [70] investigated $\mathrm{CgA}$ and catecholamine levels in postmortem serum from right heart blood and CSF of 298 forensic autopsy cases. The authors observed that most cases of hyperthermia presented higher serum and lower CSF $\mathrm{CgA}$ levels. Additionally, a marked positive correlation between $\mathrm{CgA}$ and catecholamine serum levels was detected for hyperthermia cases, which also presented a high serum/CSF $\mathrm{CgA}$ ratio and a high hypothalamus neuronal $\mathrm{CgA}$ immunopositivity, together with no change in $\mathrm{CgA}$ immunopositivity in adrenal medulla. According to the authors, these findings indicated an activation of the sympathetic/adrenomedullary system due to hyperthermic stress. However, since no relationship was detected between serum $\mathrm{CgA}$ levels and immunopositivity in adrenal medulla, the authors postulated that other sources of $\mathrm{CgA}$ and catecholamines circulating in blood could be involved in hyperthermic stress. CgA levels in CSF were low in hyperthermia cases despite prolonged death accompanied by a significant elevation in postmortem serum $\mathrm{CgA}$ levels. According to the authors, this situation could be interpreted as the consequence of brain dysfunction during systemic multiple organ failure due to hyperthermia. Hence, they postulated that $\mathrm{CgA}$ may be an independent marker of lasting stress due to environmental thermal hazards. Quan et al. [93] investigated serotonin (5-HT) levels in cerebrospinal and pericardial fluids with regard to the cause of death in 351 medicolegal autopsy cases. The authors observed that hyperthermia fatalities presented higher 5-HT levels in both fluids, suggesting hyperthermic neuronal dysfunction involving serotonin toxicity, which is characterized by neuromuscular excitation, autonomic stimulation, and an altered mental state. $\mathrm{Li}$ et al. [94] evaluated S100B levels in postmortem serum from right heart blood and cerebrospinal fluid with regard to the cause of death in 216 medicolegal autopsy cases. They observed that S100B levels in CSF were higher in hyperthermia cases and proposed a value of $2,000 \mathrm{ng} / \mathrm{ml}$ in the early postmortem period as a biochemical sign of fatally severe brain damage. 


\section{C-reactive protein and neopterin}

Fujita et al. [95] investigated C-reactive protein (CRP) levels in postmortem serum obtained from right cardiac blood in 408 medicolegal autopsy cases, including 216 cases of acute death ( $<30 \mathrm{~min}$ ) and 192 cases of non-acute death ( $>30 \mathrm{~min})$. Hyperthermia fatalities were classified as non-acute deaths and short-term survivors (survival time within $6 \mathrm{~h}$ ) and revealed low CRP levels $(<0.5 \mathrm{mg} / \mathrm{dl})$ when compared with other causes of death. Maeda et al. [96] analyzed urea nitrogen, creatinine, and CRP in postmortem serum from the external iliac vein (or right heart chamber when peripheral blood was not available) in 429 medicolegal autopsy cases. Most hyperthermia fatalities revealed low urea nitrogen and CRP levels and a markedly increased creatinine value $(>2 \mathrm{mg} / \mathrm{dl})$, with urea nitrogen-to-creatinine ratio significantly lower when compared with other studied groups that displayed elevated creatinine levels. The authors concluded that increased postmortem serum creatinine levels in hyperthermia fatalities could be the consequence of mild muscle damage, antecedent to rhabdomyolysis, or the result of systemic skeletal muscle damage in advanced multiple organ dysfunction in the absence of significant inflammation. Ishikawa et al. [97] studied the concentrations of CRP and neopterin in postmortem serum from blood obtained from different sampling sites (subclavian and external iliac veins and left and right heart). The authors observed low CRP levels $(<1 \mathrm{mg} / \mathrm{dl})$ and moderate increases (50-200 nmol/l) in neopterin concentrations in hyperthermia fatalities, suggesting that in cases with survival times $<24 \mathrm{~h}$, moderate increases in neopterin levels could be the result of a rapid activation of macrophages due to tissue injury.

\section{Tryptase}

Nishio and Suzuki [98] quantified tryptase values in postmortem serum of 122 forensic autopsy cases. In three of them, the cause of death was attributed to fatal hyperthermia, and tryptase levels were remarkably increased. Interestingly, one of these cases had been treated with neuroleptics for several years and another with clomipramine and fluvoxamine. The authors postulated that neuroleptic malignant syndrome and serotonin syndrome could be responsible for these deaths. Additionally, both cases presented high myoglobin immunoreactivity in the renal tubules, which corroborated hyperthermia as the cause of death. At the same time, the authors reported two other cases of hyperthermia that were likely induced by antidepressant or neuroleptic drugs, without elevation of serum tryptase.

\section{Myoglobin}

Zhu et al. [99] investigated urinary Mb levels in 295 forensic autopsy cases and compared them to postmortem serum levels. Increased serum $\mathrm{Mb}$ values were observed in hyperthermia fatalities, which also revealed moderate to high increases of $\mathrm{Mb}$ in urine. However, urinary $\mathrm{Mb}$ levels did not correlate with serum concentrations nor did the severity of muscle injury. Factors indicated as potentially responsible for increased urinary $\mathrm{Mb}$ levels included autolysis of the bladder wall muscle tissue $48 \mathrm{~h}$ after death and prolonged survival time associated with hypoxic muscle damage or catabolic muscle changes. According to the authors, these results suggested that high urinary myoglobin levels in acute and subacute deaths could indicate massive skeletal muscle damage due to heat stroke.

\section{Procalcitonin}

Heat stroke shares clinical similarities with other systemic inflammatory response syndromes (SIRS), being characterized by a wide spectrum of metabolic alterations and multiple organ failure. In some forms of SIRS, such as burns, severity and mortality have been clinically predicted by the greatly increased concentration of circulating procalcitonin (PCT) [100]. Some clinical studies [100-103] investigated the potential correlation between the presence and course of classic heat stroke and circulating PCT levels. Nylén et al. [100] observed significantly increased PCT serum levels in patients admitted to hospital who survived classic heat stroke. Conversely, among those who died, PCT concentrations were not elevated on admission and failed to increase further, likely suggesting that more significant increases in body heat or duration of heat exposure in patients who died may adversely affect the constitutively secreted PCT or, alternatively, upregulate enzymes that degrade secreted PCT. In a subsequent study performed by Hausfater et al. [102], PCT serum levels did not correlate with mortality, and the authors did not confirm the conclusions of Nylén et al. PCT levels appeared rather to be an indicator of illness severity and were observed in cases of heat stroke without any concomitant documented bacterial infection, suggesting that heat stroke represents a model of non-septic pathway of PCT synthesis. In agreement with the conclusions of Hausfater et al., Tong et al. [103] found that elevated PCT levels in exertional heat stroke did not indicate concomitant bacterial infections but were instead associated with the severity of the disease.

\section{Conclusions}

The aim of this article was to review the literature pertaining to biochemical investigations that can be performed in the postmortem diagnosis of heat-related fatalities (Table 1). In the absence of typical morphological findings, such a diagnosis essentially remains a medley of observations and only the evidence of exposure to high ambient temperature, as 


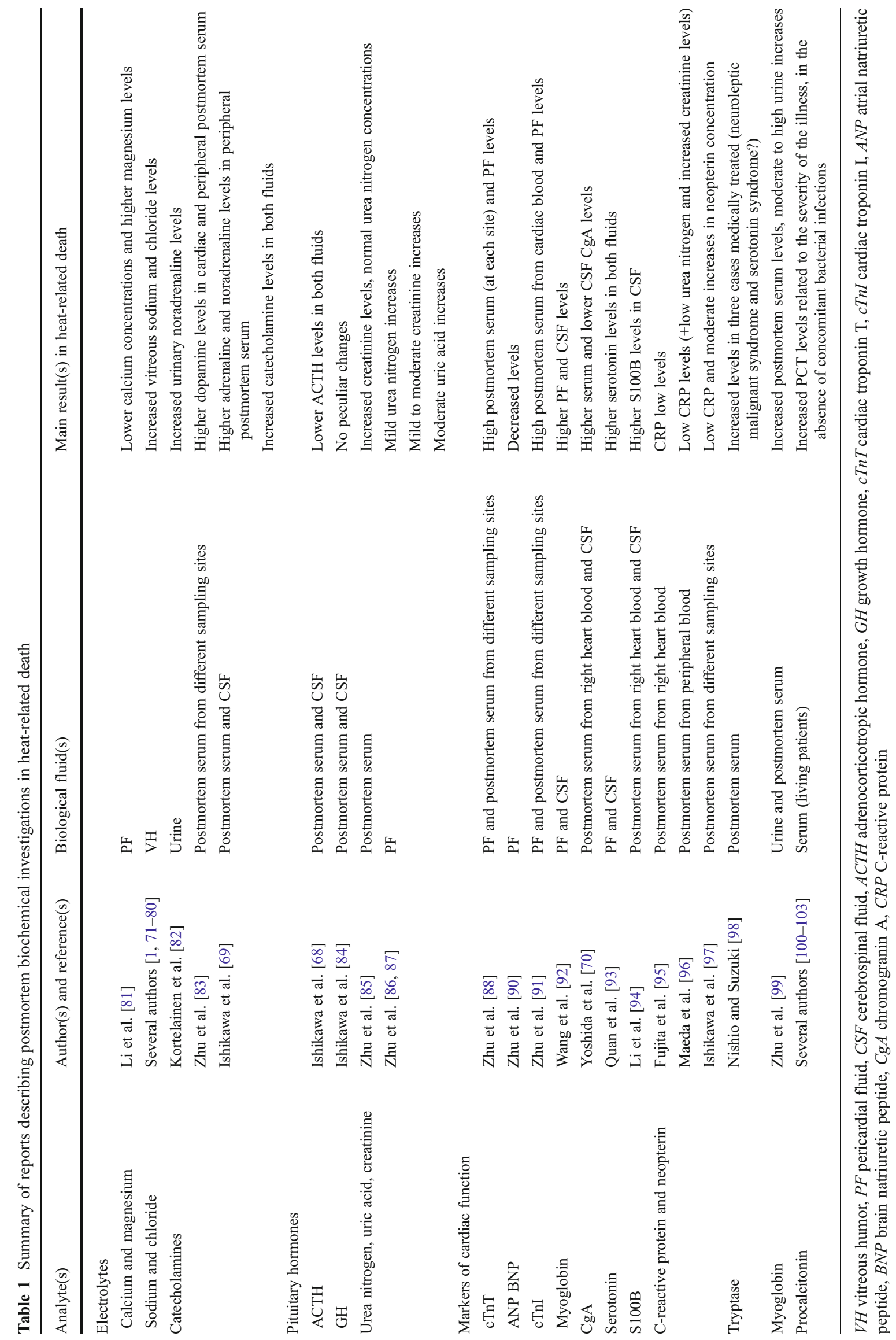


determined by investigative information, can provide elucidatory elements.

Postmortem examinations are important in order to rule out other possible causes of death rather than to establish the actual cause of death. Though the diagnosis of heat-related death remains principally based on investigative findings, radiology, autopsy, neuropathology, histology, toxicology, and biochemistry should always be carried out to exclude other causes of death.

Heat stroke is a rare disease in Europe and extensive, medicolegal studies in this area are not current. Increased vitreous sodium and chloride, postmortem serum and pericardial fluid creatinine as well as urine myoglobin are the main biochemical markers that have been traditionally observed in the postmortem analyses of heat-related fatalities. These findings are the consequences of dehydration and skeletal muscle damage, distinctive features of heat stroke. Clinically, increased PCT levels have been related to illness severity in the absence of simultaneously documented bacterial infections. These analyses should be considered a basic paradigm to perform when heat-related fatalities are suspected.

Findings concerning other postmortem biochemical markers can reflect nonspecific situations, such as the magnitude of the physical stress response and severity of myocardial damage, or may be the consequence of preexisting diseases. Such results are not constantly associated with heat-related fatalities and are thereby of little or no help in such diagnosis.

Another limit of postmortem biochemistry results for the diagnosis of heat-related fatalities is that most investigations concerning specific molecules have only been pursued by certain groups of authors whose results have never been questioned, validated, confirmed, or invalidated. Furthermore, some molecules are not routinely investigated in most medicolegal centers, so their detection in postmortem biological fluids is considered an isolated finding at present instead of a currently available investigation method.

We are of the opinion that the diagnosis of heat-related fatalities is a medley of investigative and medicolegal observations and cannot be based on postmortem biochemical analyses. Moreover, the results of the mentioned biochemical investigations must necessarily be confirmed by targeted studies on larger, heat-related death samples. However, within these limits, postmortem biochemical analyses should be performed in order to improve the quality of forensic autopsies and create reliable databases. This is particularly necessary in situations such as classic and exertional heat strokes which are relatively infrequent in forensic pathology routine and for which pathognomonic diagnostic elements are currently unavailable.

Acknowledgments The authors gratefully acknowledge the assistance of the editors and anonymous reviewers of the journal for their comments and suggestions on an earlier draft.

\section{References}

1. Nixdorf-Miller A, Hunsaker DM, Hunsaker JC 3rd (2006) Hypothermia and hyperthermia medicolegal investigation of morbidity and mortality from exposure to environmental temperature extremes. Arch Pathol Lab Med 130:1297-1304

2. Dolinak D, Matshes E, Lew E (2005) Hyperthermia. In: Forensic pathology. Principles and practice. Elsevier Academic Press, 1 ed. San Diego pp 247-248

3. Mirchandani HG, McDonald G, Hood IC, Fonseca C (1996) Heat-related deaths in Philadelphia-1993. Am J Forensic Med Pathol 17:106-108

4. Semenza JC, Rubin CH, Falter KH, Selanikio JD, Flanders WD, Howe HL, Wilhelm JL (1996) Heat-related deaths during the July 1995 heat wave in Chicago. N Engl J Med 335:84-90

5. Kaiser R, Rubin CH, Henderson AK, Wolfe MI, Kieszak S, Parrott CL, Adcock M (2001) Heat-related death and mental illness during the 1999 Cincinnati heat wave. Am J Forensic Med Pathol 22:303-307

6. Wolfe MI, Kaiser R, Naughton MP, Mirabelli MC, Yoon SS, Hanzlick R, Henderson AK (2001) Heat-related mortality in selected United States cities, summer 1999. Am J Forensic Med Pathol 22:352-357

7. Naughton MP, Henderson A, Mirabelli MC, Kaiser R, Wilhelm JL, Kieszak SM, Rubin CH, McGeehin MA (2002) Heat-related mortality during a 1999 heat wave in Chicago. Am J Prev Med 22:221-227

8. Vandentorren S, Bretin P, Zeghnoun A, Mandereau-Bruno L, Croisier A, Cochet C, Ribéron J, Siberan I, Declercq B, Ledrans M (2006) August 2003 heat wave in France: risk factors for death of elderly people living at home. Eur J Public Health 16:583-591

9. Kortelainen ML (1991) Hyperthermia deaths in Finland in 197086. Am J Forensic Med Pathol 12:115-118

10. Green H, Gilbert J, James R, Byard RW (2001) An analysis of factors contributing to a series of deaths caused by exposure to high environmental temperatures. Am J Forensic Med Pathol 22:196-199

11. Bunai Y, Akaza K, Jiang WX, Nagai A (2008) Fatal hyperthermia associated with excited delirium during an arrest. Led Med (Tokyo) 10:306-309

12. Siegler RW (1998) Fatal heatstroke in a young woman with previously undiagnosed Hashimoto's thyroiditis. J Forensic Sci 43:1237-1240

13. Chao TC, Sinniah R, Pakiam JE (1981) Acute heat stroke deaths. Pathology 13:145-156

14. Assia E, Epstein Y, Shapiro Y (1985) Fatal heatstroke after a short march at night: a case report. Aviat Space Environ Med 56:441-442

15. Shapiro Y, Seidman DS (1990) Field and clinical observation of exertional heat stroke patients. Med Sci Sports Exerc 22:6-14

16. Porter AM (1993) Heat illness and soldiers. Mil Med 158:606-609

17. Hiss J, Kahana T, Kugel C, Epsteil Y (1994) Fatal classic and exertional heat stroke-report of four cases. Med Sci Law 34:339-343

18. Gardner JW, Kark JA (1994) Fatal rhabdomyolysis presenting as mild heat illness in military training. Mil Med 159:160-163

19. Kark JA, Burr PQ, Wenger CB, Gastaldo E, Gardner JW (1996) Exertional heat illness in marine corps recruit training. Aviat Space Environ Med 67:354-360

20. Gardner JW, Kark JA, Karnei K, Sanborn JS, Gastaldo E, Burr P, Wenger CB (1996) Risk factors predicting exertional heat illness in male marine corps recruits. Med Sci Sports Exerc 28:939-944

21. Epstein Y, Moran DS, Shapiro Y, Sohar E, Shemer J (1999) Exertional heat stroke: a case series. Med Sci Sports Exerc 31:224-228 
22. Epstein Y, Shani Y, Moran DS, Shapiro Y (2000) Exertional heat stroke - the prevention of a medical emergency. J Basic Clin Physiol Pharmacol 11:395-401

23. Pretzlaff RK (2002) Death of an adolescent athlete with sickle cell trait caused ba exertional heat stroke. Pediatr Crit Care Med 3:208-210

24. Gardner JW, Gutmann FD, Potter RN, Kark JA (2002) Nontraumatic exercise-related deaths in the U.S. military, 1996-1999. Mil Med 167:964-970

25. Porter AM (2003) Collapse from exertional heat illness: implications and subsequent decisions. Mil Med 168:76-81

26. Wallace RF, Kriebel D, Punnett L, Wegman DH, Wenger CB, Gardner JW, Gonzalez RR (2005) The effects of continuous hot weather training on risk of exertional heat illness. Med Sci Sports Exerc 11:263-265

27. Byard RW, Riches KJ (2005) Dehydration and heat-related death: sweat lodge syndrome. Am J Forensic Med Pathol 26:236-239

28. Roberts KB, Roberts EC (1976) The automobile and heat stress. Pediatrics 58:101-104

29. Wadlington WB, Tucker AL Jr, Fly F, Greene HL (1976) Heat stroke in infancy. Am J Dis Child 130:1250-1251

30. Bacon C, Scott D, Jones P (1979) Heatstroke in well-wrapped infants. Lancet 24:422-425

31. Goodyear JE (1979) Heat hyperpyrexia in an infant (a case report). Med Sci Law 19:208-209

32. King K, Negus K, Vance JC (1981) Heat stress in motor vehicles: a problem in infancy. Pediatrics 68:579-582

33. Surpure JS (1982) Heat-related illness and automobile. Ann Emerg Med 11:263-265

34. Bacon CJ (1983) Over heating in infancy. Arch Dis Child 58:673-674

35. Krous HF, Nadeau JM, Fukumoto RI, Blackbourne BD, Byard RW (2001) Environmental hyperthermic infant and early childhood death: circumstances, pathologic changes, and manner of death. Am J Forensic Med Pathol 22:374-382

36. Schuliar Y, Savourey G, Besnard Y, Launey JC (2001) Diagnosis of heat stroke in forensic medicine. Contribution of thermophysiology. Forensic Sci Int 124:205-208

37. McLaren C, Null J, Quinn J (2005) Heat stress from enclosed vehicles: moderate ambient temperatures cause significant temperature rise in enclosed vehicles. Pediatrics 116:109-112

38. Guard A, Gallagher SS (2005) Heat related deaths to young children in parked cars: an analysis of 171 fatalities in the United States, 1995-2002. Inj Prev 11:33-37

39. Booth JN 3rd, Davis GG, Waterbor J, McGwin G Jr (2010) Hyperthermia deaths among children in parked vehicles: ana analysis of 231 fatalities in the United States, 1999-2007. Forensic Sci Med Pathol 6:99-105

40. Grundstein A, Null J, Meentemeyer V (2011) Weather, geography, and vehicle-related hyperthermia in children. Geogr Rev 101:353-370

41. Ohshima T, Maeda H, Takayasu T, Fujioka Y, Nakaya T (1992) An autopsy case of infant death due to heat stroke. Am J Forensic Med Pathol 13:217-221

42. Fineschi V, D'Errico S, Neri M, Pararese F, Ricci PA, Turillazzi E (2005) Heat stroke in an incubator: an immunohistochemical study in a fatal case. Int J Legal Med 119:94-97

43. Zhou Y, Li L, Liu L, Jia D, Zhang X, Fowler DR, Xu X (2006) Heat stroke deaths caused by electric blanket: case report and review of the literature. Am J Forensic Med Pathol 27:324-327

44. Chan TC, Evans DS, Clark RF (1997) Drug-induced hyperthermia. Crit Care Clin 13:785-808

45. Lannas PA, Pachar JV (1993) A fatal case of neuroleptic malignant syndrome. Med Sci Law 33:86-88

46. Fujitani N, Koda Y, Okamura T, Hattori H, Kimura H (2000) A fatal case of hyperthermia due to tricyclic antipdepressant intoxication. Leg Med (Tokyo) 2:152-153
47. Stanfield SC, Privette T (2000) Neuroleptic malignant syndrome associated with olanzapine therapy: a case report. J Emerg Med 19:355-357

48. Kubo S, Orihara Y, Kitamura O, Ikematsu K, Tsuda R, Nakasono I (2001) An autopsy case of neuroleptic malignant syndrome (NMS) and its immunohistochemical findings of muscleassociated proteins and mitochondria. Forensic Sci Int 115:155158

49. Hall KL, Taylor WH, Ware MR (2001) Neuroleptic malignant syndrome due to olanzapine. Psychopharmacol Bull 35:49-54

50. Sheil AT, Collins KA, Schandl CA, Harley RA (2007) Fatal neurotoxic response to neuroleptic medications. Case report and review of the literature. Am J Forensic Med Pathol 28:116-120

51. Kemp WL, Fitzgerald J, White CL 3rd (2007) Undiagnosed progressive supranuclear palsy in a patient with neuroleptic malignant syndrome due to use of neuroleptics: the utility of autopsy in deaths due to known drug reactions. Am J Forensic Med Pathol 28:59-62

52. Patil PM (2011) Malignant hyperthermia in the oral and maxillofacial surgery patient: un update. Oral Surg Oral Med Oral Pathol Radiol Endod 11:1-7

53. Ali SZ, Taguchi A, Rosenberg H (2003) Malignant hyperthermia. Best Pract Res Clin Anaesthesiol 17:519-533

54. Nishio H, Sato T, Fukunishi S, Tamura A, Iwata M, Tsuboi K, Suzuki K (2009) Identification of malignant hyperthermiasusceptible ryanodine receptor type 1 gene (RYR1) mutations in a child who died in a car after exposure to a high environmental temperature. Leg med (Tokyo) 11:142-143

55. Sato T, Nishio H, Iwata M, Tsuboi K, Tamura A, Miyazaki T, Suzuki K (2010) Postmortem molecular screening for mutations in ryanodine receptor type 1 (RYR1) gene in psychiatric patients suspected of having died of neuroleptical malignant syndrome. Forensic Sci Int 194:77-79

56. Sternbach H (1991) The serotonin syndrome. Am J Psychiatry 148:705-713

57. Bodner RA, Lynch T, Lewis L, Kahn D (1995) Serotonin syndrome. Neurology 45:219-223

58. Mueller PD, Korey WS (1998) Death by "Ecstasy": the serotonin syndrome? Ann Emerg Med 32:377-380

59. Vuori E, Henry JA, Ojanperä I, Nieminen R, Savolainen T, Wahlsten P, Jäntti M (2003) Death following ingestion of MDMA (Ecstasy) and moclobemide. Addiction 98:365-368

60. Warrick BJ, Wilson J, Hedge M, Freeman S, Leonard K, Aaron C (2011) Lethal serotonin syndrome after methylone and butylone ingestion. J Med Toxicol. doi:10.1007/s13181-011-0199-6

61. Lifschultz BD, Donoghue ER (1998) Forensic pathology of heatand cold-related injuries. Clin Lab Med 18:77-90

62. Donoghue ER, Graham MA, Jentzen JM, Lifschutz BD, Luke JL, Mirchandani HG (1997) Criteria for the diagnosis of heat-related death: National Association of Medical Examiners. Position paper. National Association of Medical Examiners Ad Hoc Committee on the definition of heat-related fatalities. Am J Forensic Med Pathol 18:11-14

63. Sweeney KG (2002) Heat-related deaths. J Insur Med 34:114 119

64. Inoue H, Ikeda N, Tsuji A, Kudo K, Hanagama M, Nata M (2009) Pulmonary fat embolization as a diagnostic finding for heat exposure. Led Med (Tokyo) 11:1-3

65. Quan L, Ishikawa T, Michiue T, Li DR, Zhao D, Zhu BL, Maeda $\mathrm{H}$ (2005) Quantitative analysis of ubiquitin-immunoreactivity in the midbrain periaqueductal gray matter with regard to the cause of death in forensic autopsy. Leg Med (Tokyo) 7:151-156

66. Ishikawa T, Zhu BL, Li DR, Zhao D, Michiue T, Maeda H (2007) Immunohistochemical investigation of ubiquitin and myoglobin in the kidney in medicolegal autopsy cases. Forensic Sci Int 171:136-141 
67. Smith HJ, Khal J, Tisdale MJ (2005) Downregulation of ubiquitin-dependent protein degradation in murine myotubes during hyperthermia by eicosa pentaenoic acid. Biochem Biophys Res Commun 332:83-88

68. Ishikawa T, Quan L, Li DR, Zhao D, Michiue T, Hamel M, Maeda H (2008) Postmortem biochemistry and immunohistochemistry of adrenocorticotropic hormone with special regard to fatal hypothermia. Forensic Sci Int 179:147-151

69. Ishikawa T, Yoshida C, Michiue T, Perdekamp MG, Pollak S, Maeda H (2010) Immunohistochemistry of catecholamines in the hypothalamic-pituitary-adrenal system with special regard to fatal hypothermia and hyperthermia. Leg Med (Tokyo) 12:121-127

70. Yoshida C, Ishikawa T, Michiue T, Quan L, Maeda H (2011) Postmortem biochemistry and immunohistochemistry of chromogranin A as a stress marker with special regard to fatal hypothermia and hyperthermia. Int J Legal Med 125:11-20

71. Maeda H, Ishikawa T, Michiue T (2011) Forensic biochemistry for functional investigation of death: concept and practical application. Leg Med (Tokyo) 13:55-67

72. Palmiere C, Mangin M (2011) Postmortem chemistry update part I. Int J Legal Med 126:187-198

73. Coe JI (1993) Postmortem chemistry update. Emphasis on forensic application. Am J Forensic Med Pathol 14:91-117

74. Thierauf A, Musshoff F, Madea B (2009) Postmortem biochemical investigations of vitreous humor. Forensic Sci Int 192:78-82

75. Coe JI (1969) Postmortem chemistries on human vitreous humor. Am J Clin Pathol 51:741-750

76. Coe JI (1972) Use of chemical determinations on vitreous humor in forensic pathology. J Forensic Sci 17:541-546

77. Coe JI (1974) Postmortem chemistry: practical considerations and a review of the literature. J Forensic Sci 19:13-32

78. Coe JI (1977) Postmortem chemistry of blood, cerebrospinal fluid, and vitreous humor. In: Tedeschi CG, Eckert WG, Tedeschi LG (eds) Forensic medicine, vol 2. Saunders, Philadelphia, pp 1030-1060

79. Coe JI (1977) Postmortem chemistry of blood, cerebrospinal fluid, and vitreous humor. Leg Med Annu 1976:55-92

80. Ingham AI, Byard RW (2009) The potential significance of elevated vitreous sodium levels at autopsy. J Forensic Leg Med $16: 437-440$

81. Li DR, Quan L, Zhu BL, Ishikawa T, Michiue T, Zhao D, Yoshida C, Chen JH, Wang Q, Komatsu A, Azuma Y, Maeda H (2009) Evaluation of postmortem calcium and magnesium levels in the pericardial fluid with regard to the cause of death in medicolegal autopsy. Leg Med (Tokyo) 11:S276-S278

82. Kortelainen ML, Huttunen P, Lipinlampi T (1990) Urinary catecholamines in hyperthermia-related deaths. Forensic Sci Int 48:103-110

83. Zhu BL, Ishikawa T, Michiue T, Li DR, Zhao D, Quan L, Oritani S, Bessho Y, Maeda H (2007) Postmortem serum catecholamine levels in relation to the cause of death. Forensic Sci Int 173:122-129

84. Ishikawa T, Michiue T, Maeda H (2011) Evaluation of postmortem serum and cerebrospinal fluid growth hormone levels in relation to the cause of death in forensic autopsy. Hum Cell 24:74-77

85. Zhu BL, Ishida K, Quan L, Taniguchi M, Oritani S, Li DR, Fujita MQ, Maeda H (2002) Postmortem serum uric acid and creatinine levels in relation to the causes of death. Forensic Sci Int 125:59-66

86. Zhu BL, Ishikawa T, Michiue T, Li DR, Zhao D, Quan L, Maeda H (2005) Evaluation of postmortem urea nitrogen, creatinine and uric acid levels in pericardial fluid in forensic autopsy. Leg Med (Tokyo) 7:287-292

87. Zhu BL, Ishikawa T, Michiue T, Tanaka S, Zhao D, Li DR, Quan L, Oritani S, Maeda H (2007) Differences in postmortem urea nitrogen, creatinine and uric acid levels between blood and pericardial fluid in acute death. Leg Med (Tokyo) 9:115-122
88. Zhu BL, Ishikawa T, Michiue T, Li DR, Zhao D, Oritani S, Kamikodai Y, Tsuda K, Okazaki S, Maeda H (2006) Postmortem cardiac troponin T levels in the blood and pericardial fluid. Part 1. Analysis with special regard to traumatic causes of death. Leg Med (Tokyo) 8:86-93

89. Zhu BL, Ishikawa T, Michiue T, Li DR, Zhao D, Kamikodai Y, Tsuda K, Okazaki S, Maeda H (2006) Postmortem cardiac troponin $\mathrm{T}$ levels in the blood and pericardial fluid. Part 2: analysis for application in the diagnosis of sudden cardiac death with regard to pathology. Leg Med (Tokyo) 8:94-101

90. Zhu BL, Ishikawa T, Michiue T, Li DR, Zhao D, Tanaka S, Kamikodai Y, Tsuda K, Okazaki S, Maeda H (2007) Postmortem pericardial natriuretic peptides as markers of cardiac function in medico-legal autopsies. Int J Legal Med 121:28-35

91. Zhu BL, Ishikawa T, Michiue T, Li DR, Zhao D, Bessho Y, Kamikodai Y, Tsuda K, Okazaki S, Maeda H (2007) Postmortem cardiac troponin I and creatine kinase MB levels in the blood and pericardial fluid as markers of myocardial damage in medicolegal autopsy. Leg Med (Tokyo) 9:241-250

92. Wang Q, Michiue T, Ishikawa T, Zhu BL, Maeda H (2011) Combined analyses of creatine kinase MB, cardiac troponin I and myoglobin in pericardial and cerebrospinal fluids to investigate myocardial and skeletal muscle injury in medico-legal autopsy cases. Leg Med (Tokyo) 13:226-232

93. Quan L, Ishikawa T, Hara J, Michiue T, Chen JH, Wang Q, Zhu BL, Maeda H (2011) Postmortem serotonin levels in cerebrospinal and pericardial fluids with regard to the cause of death in medicolegal autopsy. Leg Med (Tokyo) 13:75-78

94. Li DR, Michiue T, Zhu BL, Ishikawa T, Quan L, Zhao D, Yoshida C, Chen JH, Wang Q, Komatsu A, Azuma Y, Maeda H (2009) Evaluation of postmortem S100B levels in the cerebrospinal fluid with regard to the cause of death in medicolegal autopsy. Leg Med (Tokyo) 11:S273-S275

95. Fujita MQ, Zhu BL, Ishida K, Quan L, Oritani S, Maeda H (2002) Serum C-reactive protein levels in postmortem blood-an analysis with special reference to the cause of death and survival time. Forensic Sci Int 130(2-3):160-166

96. Maeda H, Zhu BL, Bessho Y, Ishikawa T, Quan L, Michiue T, Zhao D, Li DR, Komatsu A (2008) Postmortem serum nitrogen compounds and C-reactive protein levels with special regard to investigation of fatal hyperthermia. Forensic Sci Med Pathol 4:175-180

97. Ishikawa T, Hamel M, Zhu BL, Li DR, Zhao D, Michiue T, Maeda H (2008) Comparative evaluation of postmortem serum concentrations of neopterin and C-reactive protein. Forensic Sci Int 179:135-143

98. Nishio H, Suzuki K (2005) Three cases of suspected hyperthermia with remarkable elevation of serum mast cell tryptase. Forensic Sci Int 149:51-55

99. Zhu BL, Ishida K, Quan L, Taniguchi M, Oritani S, Kamikodai Y, Fujita MQ, Maeda H (2001) Post-mortem urinary myoglobin levels with reference to the cause of death. Forensic Sci Int 115:183-188

100. Nylén ES, Al Arifi A, Becker KL, Snider RH Jr, Alzeer A (1997) Effect of classic heatstroke on serum procalcitonin. Crit Care Med 25:1362-1365

101. Oberhoffer M, Karzai W, Meier-Hellmann A, Reinhart K (1998) Procalcitonin. A new diagnostic parameter for severe infections and sepsis. Anaesthesist 47:581-587

102. Hausfater P, Hurtado M, Pease S, Juillien G, Lvovschi VE, Salehabadi S, Lidove O, Wollf M, Bernard M, Chollet-Martin $\mathrm{S}$, Riou B (2008) Is procalcitonine a marker of critical illness in heatstroke? Intensive Care Med 34:1377-1383

103. Tong HS, Liu YS, Wen Q, Tang YQ, Yuan FF, Su L (2012) Serum procalcitonin predicting mortality in exertional heatstroke. Emerg Med J 29:113-117 